\title{
Le playwork à la recherche d'une identité perdue
}

Playwork - in search of an identity

\section{Shelly Newstead}

Traducteur : Baptiste Besse-Patin et Nathalie Roucous

\section{(2) OpenEdition \\ Journals}

Édition électronique

URL : https://journals.openedition.org/sdj/2337

DOI : $10.4000 /$ sdj.2337

ISSN : 2269-2657

\section{Éditeur}

Laboratoire EXPERICE - Centre de Recherche Interuniversitaire Expérience Ressources Culturelles Education

\section{Référence électronique}

Shelly Newstead, "Le playwork à la recherche d'une identité perdue », Sciences du jeu [En ligne], 12 I 2019, mis en ligne le 19 décembre 2019, consulté le 12 décembre 2022. URL : http:// journals.openedition.org/sdj/2337 ; DOI : https://doi.org/10.4000/sdj.2337

Ce document a été généré automatiquement le 12 décembre 2022.

\section{(c) (i) (9)}

Creative Commons - Attribution - Pas d'Utilisation Commerciale - Pas de Modification 4.0 International - CC BY-NC-ND 4.0

https://creativecommons.org/licenses/by-nc-nd/4.0/ 


\title{
Le playwork à la recherche d'une identité perdue
}

\author{
Playwork - in search of an identity
}

\section{Shelly Newstead}

Traduction : Baptiste Besse-Patin et Nathalie Roucous

Plus de soixante-dix ans après l'ouverture du premier terrain d'aventure au Danemark, le playwork $^{1}$ et les terrains d'aventure connaissent actuellement une (sorte de) renaissance théorique et pratique à l'échelle mondiale. De nouveaux terrains d'aventure et des projets associés ont émergé dans de nombreux pays (Milne, 2014 ; Leichter-Saxby \& Law, 2015). On constate également une explosion du nombre des publications autour de cette thématique avec des ouvrages dédiés ou des chapitres d'ouvrage écrits par des spécialistes rendant cette pensée accessible à un plus large public (Maclean, Russell \& Ryall, 2015 ; Kilvington \& Wood, 2010). La recherche s'est aussi considérablement étoffée ces dernières années, avec la publication par ceux qui œuvrent dans le domaine d'articles de revues et de textes scientifiques dans diverses revues académiques à l'auditoire varié (Waters, 2014; van Rooijen \& Newstead, 2016 ; Wragg, 2016). De plus, les réseaux sociaux ont facilité sa diffusion au grand public comme jamais dans son histoire. Les professionnelle-s et les parents de tous les coins du monde peuvent se tourner vers la pensée et les pratiques portées par ce mouvement pour une approche alternative du travail avec les enfants (Mortenson, 2014 ; Wong, 2015), connue sous le vocable de playwork.

2 En dépit de ce renforcement du statut et de l'influence internationale, le playwork se confronte encore, au Royaume-Uni, à des défis sérieux dans sa quête d'une reconnaissance professionnelle. Pour de nombreuses personnes travaillant dans ce champ, l'établissement d'un référentiel métier national [National Occupational Standards for Playwork] au début des années 1990 a marqué le début de cette nouvelle ère de reconnaissance. Cependant, il n'est pas encore complètement admis que le playwork mérite le statut de profession (Palmer, Wilson \& Battram, 2007). Selon les quatre caractéristiques définissant une profession retenues par Banks (2010) - existence d'un code déontologique, d'associations professionnelles, de formations qualifiantes et d'une 
reconnaissance légale -, le champ britannique du playwork peine à remplir toutes les conditions. Bien que certaines avancées aient été effectuées sur la dimension éthique (Else, 2007; Stonehouse, 2015), ce travail n'a pas été repris par l'ensemble du champ professionnel. En ce qui concerne des associations professionnelles, au cours des soixante-dix dernières années, de nombreuses tentatives ont été menées en vue de la création de structures nationales, qu'il s'agisse de syndicats ou d'organismes de formation et de soutien à l'emploi subventionnés par le gouvernement ou encore d'organismes professionnels locaux (Shier, 1996; SkillsActive, 2006). Pour autant, toutes ont été de courte durée ou ne fonctionnent qu'avec très peu de membres. Au cours de la dernière décennie, le nombre de formations professionnelles a diminué à tous les niveaux de qualification, la majorité d'entre elles ayant été interrompues par manque de participantees (Dallal, 2015 ; McKendrick et alii, 2015). Bien qu'il n'existe pas de chiffres fiables permettant d'évaluer avec précision l'ampleur du phénomène (King et Newstead, 2017), on peut - sans risque - faire l'hypothèse qu'une diminution de l'offre de formations a entraîné une diminution du nombre de professionnelle·s formée's et qualifiée's. Au mieux, la reconnaissance légale de la profession apparaît donc comme très inégalement répartie sur le territoire du Royaume-Uni, les playworkers étant souvent reconnus en fonction du cadre dans lequel ils travaillent, plutôt qu'en tant que professionnellle's à part entière. En fait, ce qui apparaît, de l'extérieur, comme une profession établie au Royaume-Uni, s'avère être davantage une communauté de pratique (Lave \& Wenger 1991) vivant avec un espoir d'évolution.

Cette contribution explore un des problèmes fondamentaux qui sous-tend l'absence de statut et de reconnaissance professionnelle du playwork: la question de l'identité. Elle cherche à montrer que la reconnaissance d'un statut professionnel est conditionnée à une définition cohérente et consensuelle de ce que signifie la pratique du playworker. Le texte commence par décrire comment le playwork a été inventé comme une approche alternative du travail avec les enfants à partir des terrains d'aventure britanniques créés juste après la Seconde Guerre mondiale, puis comment il a été professionnalisé dans les années 1990. Il se poursuit en décrivant comment il a été défini de diverses façons au fil des années, pour évoquer les questions et les défis soulevés par ces définitions. Enfin, la conclusion tire les leçons de l'histoire du playwork qui peuvent contribuer à son développement en tant que profession à l'échelle internationale.

Cet article repose sur une recherche documentaire utilisant la littérature historique sur les terrains d'aventure réalisée dans le cadre d'une recherche doctorale sur les origines du playwork (Newstead, 2016). Il est communément admis que la littérature produite par les pionniers des terrains d'aventure est soit inexistante, soit extrêmement limitée (Brown, Long \& Wragg, 2018). Cependant, la recherche doctorale a permis d'identifier près de 500 sources primaires historiques, c'est-à-dire écrites par un auteur ayant une expérience directe de travail dans les terrains d'aventure ou soutenant leur développement. Ce recueil de données a été circonscrit à la littérature prenant pour objet les terrains d'aventure - tels que définis par lady Allen de Hurtwood comme précisé plus loin - et produite entre 1946, date de l'article du Picture Post dans lequel elle introduit ce type de terrain au Royaume-Uni, et 1990, période où est élaboré le référentiel national du playwork. 
Cette littérature rare et oubliée a été retrouvée à l'aide d'une stratégie de recherche systématique, qui comprenait :

- la technique de «boule de neige» (Ramer, 2005). Des citations d'une source primaire sont utilisées pour identifier d'autres documents pertinents qui, à leur tour, ont cité d'autres documents pertinents, et ainsi de suite.

- la recherche par auteur'e. Les noms des auteure's ont été recherchés pour identifier leurs productions. Comme la plupart de ces auteurs étaient inconnus, des recherches biographiques approfondies ont également été menées pour établir leur implication dans les terrains d'aventure et ainsi s'assurer de la crédibilité de leur travail comme source primaire (Tosh \& Lang, 2006).

- la recherche de mots-clés thématiques. Des termes techniques propres aux terrains d'aventure, comme playleadership ou junk playgrounds, ont été utilisés pour identifier les productions qui avaient été écrites par les pionniers, mais qui n'avaient pas été classées comme une littérature associée.

6 Les sources primaires historiques ont été identifiées au moyen de catalogues de bibliothèques en ligne, d'archives de journaux, de bases de données du monde de l'éducation, de sites de réseaux sociaux, de documents archivés libres de droits, de livres d'occasion disponibles et de documents audiovisuels historiques. Ainsi, 243 sources primaires historiques ont été consultées et analysées en suivant une démarche fondée sur la théorie ancrée [Grounded Theory] (Glaser \& Strauss, 2010). Ces sources ont été lues dans leur intégralité et un codage ouvert (Charmaz, 2003) a été utilisé afin d'éviter l'interprétation anachronique des concepts et pratiques historiques à partir d'un point de vue contemporain sur le playwork.

7 Les récits historiques du développement du playwork ont tendance à provenir de la mémoire et des expériences personnelles de certains acteurs du mouvement (Hughes, 2006; Chilton, 2013). Bien qu'ils soient précieux en tant que sources primaires, ces récits ne sont pas toujours fiables pour situer avec précision des événements historiques et ils ne rendent pas nécessairement compte des contextes pertinents plus larges, au-delà de l'expérience de l'auteur lui-même (Roberts, 2002). Ces documents ont donc été analysés avec d'autant plus de prudence.

\section{Des terrains d'aventure au playwork}

8 Ce qui est connu comme "playwork " aujourd'hui trouve son origine dans les terrains d'aventure britanniques aménagés juste après la Seconde Guerre mondiale. Ces terrains ont initialement été imaginés par l'urbaniste et architecte paysagiste danois C.T. Sørensen. Son idée originale est apparue pour la première fois dans un livre, publié uniquement en danois, intitulé Parkpolitik i Sogn og Købstad en 1931 après avoir observé des enfants jouer dans un terrain vague au lieu d'une aire de jeux voisine. Sørensen y a inventé le terme skrammellegeplads pour décrire un espace délimité qui faciliterait les comportements naturellement créatifs et spontanés des enfants dans un milieu urbanisé (Andersson \& Høyer, 2001). Comme Sørensen ne parlait ni n'écrivait l'anglais, le terme danois inventé a d'abord été traduit par «terrain de jeux avec des matériaux récupérés » [waste material playground], mais c'est le terme " terrain de jeu bric-à-brac » [junk playground] qui a été finalement adopté au Royaume-Uni dans la traduction anglaise (Sørensen, 1947). En 1943, un collègue de Sørensen, Dan Fink, utilisa ce nouveau concept pour concevoir et construire les premiers skrammellegeplads à Emdrup, 
un nouveau quartier de Copenhague (Sørensen, 1947). Après une visite impromptue de ce quartier, lady Allen de Hurtwood écrivit un article pour le magazine très populaire, à l'époque, Picture Post (1946) qui inspira le développement de ce qui sera ensuite connu sous le nom de «terrains d'aventure » [adventure playground] dans tout le Royaume-Uni.

Les skrammellegeplads ont également été développés dans plusieurs pays d'Europe à la même époque et ont reçu des noms différents selon les pays, comme «les jardins (plaines) Robinson » en Suisse ou les abenteuerspielplatz en Allemagne (Holme \& Massie, 1970; Rosati, 1975). Au niveau international, les terrains d'aventure présentaient plusieurs caractéristiques communes, comme l'approvisionnement en matériaux et objets usagés et récupérés ou la présence d'adultes, mais aussi des problèmes similaires, comme ceux du recrutement et de la formation de ces adultes (Allen de Hurtwood, 1964). Cependant, dans certains pays, une philosophie différente, évoquée par Jago (1972, p. 2) en termes d'« extension de la société adulte», a été adoptée. Mais celle-ci n'a pas séduit les pionniers britanniques qui préféraient une approche plus axée sur et orientée par l'enfant dans les terrains d'aventure (Benjamin, 1961).

Lady Allen de Hurtwood a retenu le terme de «terrain d'aventure " dans un dépliant publié par l'Association Nationale des Terrains de Jeu [National Playing Fields Association (NPFA)] (Allen de Hurtwood, 1961) et a fourni la première définition officielle pour le Oxford English Dictionary: "un terrain de jeu créatif avec des outils et des déchets." Dans A Supplement to Oxford English Dictionary (1972), une définition plus large dispose d'une entrée : « un terrain de jeu où des matériaux divers souvent usagés sont mis à la disposition des enfants avec lesquels ils peuvent s'amuser » (cité par Allen de Hurtwood \& Nicholson, 1975, p. 233).

11 Les adultes qui travaillaient sur les premiers terrains d'aventure étaient à l'origine identifiés sous le nom de "gardiens» (NPFA, 1960). Les origines de ce titre semblent obscures, mais il peut avoir été emprunté au mouvement des centres sociaux [settlement] qui a ouvert et géré plusieurs des premiers terrains d'aventure. Mais quelles que soient ses origines, ce terme a été jugé trop autoritaire au regard des visées des terrains d'aventure (Allen de Hurtwood, 1964) et le titre des adultes intervenants sur les terrains d'aventure a rapidement été changé en "meneur de jeu» [playleader] (NPFA, 1957). En référence à cette pratique, le terme playleadership s'était déjà développé aux États-Unis dès la seconde moitié du $18^{\mathrm{e}}$ siècle (Frost, 2012) avant sa diffusion en Europe. Un premier colloque consacré à ce domaine s'est tenu lieu en 1933 au Royaume-Uni (Central Council of Recreative Physical Training, 1937). À l'origine, le playleadership était associé à l'encadrement de terrains de jeu et d'équipements fixes et impliquait souvent l'organisation de jeux et d'activités par des adultes en milieu urbain (Curtis, 1915). Pour dénommer les adultes exerçant dans les terrains d'aventure, l'adoption du terme de «meneur de jeu » en remplacement de "gardien » a été, dès le début, considérée comme une solution inadaptée (Nicholson, 1954). Bon nombre de pionniers du mouvement n'étaient pas à l'aise avec les connotations de directivité adulte qui était perçue comme allant à l'encontre de la finalité même des terrains d'aventure (Lambert, 1974). Comme l'a noté Bengtsson (1975, p. 6), le rôle des adultes devait s'entendre comme étant « tout SAUF [celui d']un meneur de jeu [playleader] ».

12 C'est pourquoi le terme de "playworker » a été créé pour distinguer le rôle des adultes travaillant dans les terrains d'aventure de celui de "meneur de jeu». Avant son association avec les terrains d'aventure, le terme de playwork était déjà utilisé pour décrire, dans le langage courant, des activités non sérieuses qui ressemblaient au 
travail, auxquelles prenaient part des adultes ou des enfants (Chambers' Journal, 1884). Il avait également été utilisé dans le cadre des programmes de playleadership et dans les premiers terrains d'aventure eux-mêmes (Allen de Hurtwood, 1953). Toutefois, l'adoption du terme résulte d'un autre compromis linguistique pour ces pionniers, car ceux qui ont participé à l'abandon du terme «meneur de jeu» estimaient que le mot français «animateur" décrivait plus précisément leur rôle. Cependant, aucune traduction satisfaisante en anglais n'a pu être trouvée, de sorte que le terme "playworker» a été adopté comme la meilleure solution (International Playgrounds Association [IPA], 1975). Ainsi, le terme playworker a progressivement remplacé celui de «meneur de jeu» tout au long des années 1970, bien que ce dernier ait été encore utilisé à la fin des années 1980 (PlayEducation, 1987). Enfin, le terme de playwork semble avoir dérivé du titre d'emploi playworker afin de décrire le travail associé à ce nouveau rôle.

Depuis l'invention de ce nouveau rôle pour les adultes dans les terrains d'aventure, ceux qui travaillent dans ce qui est devenu le monde du playwork britannique ont admis la nécessité de faire reconnaître leur travail comme une approche distincte du travail avec les enfants (Allen, 1953). S'appuyant sur des sources primaires historiques et contemporaines, la section suivante explique comment cette approche alternative a tenté, au cours des 70 dernières années, d'assurer son identité et son avenir par la professionnalisation. Pour ce qui est des sources primaires de la période contemporaine, elles se limitent et se concentrent sur les auteure's qui ont eu une expérience directe de playwork entre 1990 et aujourd'hui (Newstead, 2016).

\section{La professionnalisation du playwork}

Lorsque parait l'article de lady Allen de Hurtwood $(1946)^{2}$, qui a instigué la création des terrains d'aventure à Londres puis au Royaume-Uni, il n'existait pratiquement aucune littérature publiée pour faire connaître et soutenir ces initiatives (Allen de Hurtwood, 1954). Les personnes concernées devaient partir de zéro et donc trouver comment mettre en place et gérer ces espaces peu orthodoxes pour les enfants. L'un des nombreux défis qu'ils ont dû relever a été de décrire le rôle des adultes sur les terrains d'aventure au quotidien. Il était largement reconnu que le succès d'un terrain dépendait de la présence de ce que Bengtsson (1970, p. 174) décrivait comme «le bon type » d'adulte dans ce rôle (TCCAAPC, 1955). Cependant, définir et décrire ce qui constitue un "bon type » s'est avéré être une "noix encore plus difficile à casser " (Bengtsson, 1970, p. 163). Les adultes qui ont commencé à travailler sur les terrains d'aventure avaient des profils personnels et professionnels très variés (Allen de Hurtwood \& Nicholson, 1975), et ces pionniers ont constaté que certains adultes semblaient «naturellement " bien adaptés à ce travail, contrairement à d'autres qui échouaient à endosser ce rôle particulier (Mays, 1957 ; Abernethy, 1984). Par ailleurs, ils ont constaté qu'il leur était relativement aisé de fournir une description de ce que Abernethy (1968, p. 38) appelait les "détails opérationnels" du rôle, mais ils ne parvenaient pas à présenter le travail au-delà des tâches techniques de la gestion du terrain (Nicholson, 1954 ; Crowther, 1968). Les pionniers en sont venus à croire que les adultes devaient posséder des qualités intrinsèques indéfinissables, qui allaient au-delà des critères plus concrets du cursus de formation suivi ou des compétences techniques (Mays, 1957 ; Turner, 1961). La description mémorable du profil adapté par Abernethy 
(1968) comme « une combinaison de mère et de père, de policier et de Robin des Bois " a souvent été reprise comme une façon de résumer ces qualités personnelles (Barrand, 1972 ; Geary, 1972).

À la fin des années 1970, aucune avancée n'avait été réalisée dans la description du rôle de l'adulte, si ce n'est en termes pratiques et techniques. On s'accorda pour dire qu'il était plus ou moins impossible de parvenir à une définition de ce rôle particulier (Turner, 1961). Un consensus relatif s'est dégagé pour considérer que les adultes qui trouvaient leur place dans les terrains d'aventure devaient posséder "une qualité insaisissable " (Lambert, 1974, p. 20) qui ne pouvait être définie et que les «bonnes recrues » étaient probablement "nées ainsi et ne se formaient pas " (Lambert, 1974, p. 84). Les nouvelles recrues se sont souvent retrouvées livrées à elles-mêmes puisque " on ne peut pas recommander ou enseigner une approche standardisée " (Turner, 1961, p. 171). De ce fait, « le bon type » d'adulte était généralement considéré comme celui qui parvenait à trouver seul l'approche attendue, avec en conséquence un important renouvellement du personnel sur les terrains d'aventure.

Malgré l'absence d'une description claire des pratiques adaptées, ces pionniers des terrains d'aventure étaient convaincus que leur approche devait être adoptée par d'autres professions intervenant auprès des enfants et ont cherché à la diffuser. Ainsi, des personnalités clés dans le développement de ce type de terrain ont participé à la création au niveau local de "programmes d'activités ludiques", plus connus aujourd'hui sous le nom de playschemes ${ }^{3}$ (The Rural District Council of Wellington, 1963). À la fin des années 1980, le playwork - comme on avait commencé à le désigner prenait aussi place dans une grande variété de structures, comme les hôpitaux, les centres de loisirs [playcentres ${ }^{4}$ ] et les foyers d'hébergement, et ce avec un nombre croissant d'intervenante's rémunérée's, même s'il s'agissait encore d'un effectif relativement restreint au total (Joint National Committee on Training for Playwork [JNCTP], 1985). Cependant, en l'absence de définition ou d'un référentiel de ce qu'était l'activité au-delà de la gestion d'un terrain, le sens de l'expression «faire du playwork» a été réinterprétée et réinventée par des professionnelle's de divers horizons et philosophies, tels que des enseignante's ou des intervenants sociaux. Certains playworkers craignaient pour la survie du playwork tel qu'il était pratiqué à l'origine, comme le résume King (1988): «Si nous n'établissons pas de bonnes pratiques, les pratiques instaurées ne seront pas du playwork ». Cette crainte de l'anéantissement, due à la prolifération de pratiques diversifiées, a donné lieu à une tentative semi-formalisée pour décrire les " compétences du playwork», en reprenant les descriptions du travail par les pionniers uniquement en termes de tâches et de qualités personnelles (voir National Children's Play and Recreation Unit [NCPRU], 1991).

17 Cette initiative du monde du playwork a été rapidement rattrapée par le gouvernement qui élabora un référentiel national de qualification professionnel [National Occupational Standards] (Dyke, 1997). Beaucoup de playworkers ont perçu ce nouveau cadre de référence comme une occasion d'obtenir une reconnaissance et un statut pour leur profession (NCPRU, 1991). Ainsi, le premier référentiel professionnel introduit en 1992 a été élaboré avec une contribution importante de praticien'ne's du playwork (Bonel \& Lindon, 1996). En dépit de cette contribution issue du champ, ce référentiel a toujours été critiqué par les playworkers britanniques pour avoir fourni une description uniquement en termes fonctionnels et techniques sans avoir réussi à en saisir l'« essence » (PLAYLINK, 1997; Davy, 2007). Selon eux, le référentiel appréhende le 
playwork comme un travail généraliste qui peut s'exercer avec un ensemble de compétences génériques partagées avec d'autres métiers de l'enfance, plutôt que de décrire ce qui est distinct et unique dans le travail du playworker en comparaison à d'autres professions de l'enfance (Nicholls, 2002 ; Garvey \& Lancaster, 2010).

\section{Qu'est-ce que le playwork?}

18 Il est souvent surprenant pour ceux qui s'intéressent au playwork en dehors $d u$ Royaume-Uni d'apprendre que, malgré son histoire établie et son statut " professionnel » actuel, il n'existe en fait aucune définition communément acceptée ni de description de la spécificité des pratiques.

Au cours des quarante dernières années, le champ britannique du playwork s'est enlisé dans ce que Shier (1990) a décrit diplomatiquement comme des «années de débats stériles » sur sa nature et sa finalité. La croyance des pionniers dans l'impossibilité de le définir a persisté jusque dans les années 1980 et 1990 et, elle reste vivace dans le monde britannique. La rhétorique innéiste du « né et non formé » demeure très répandue dans la littérature contemporaine où les playworkers décrivent souvent leurs qualités en termes d'intuition et de dispositions personnelles (Russell, 2005). Encore aujourd'hui, l'idée qu'il est plus facile de reconnaître un playworker «naturel » que de formuler ce qui en constitue un (Armitage, 2014) est largement répandue. Certains playworkers sont allés jusqu'à affirmer que le playwork ne devrait pas être défini, car cela détruirait son essence même (Wilson, 2008a). Cependant, d'autres ont soutenu que cette absence de définition était contre-productive empêchant, d'une part, le playwork d'être pris au sérieux par le gouvernement, les financeurs et le grand public et, d'autre part de consolider son statut pour soutenir la crédibilité de cette approche alternative du travail avec les enfants (Balmforth, 1982; Williams, 1986). Les playworkers ont été constamment frustrés par la perception de l'opinion publique de leur travail qui consiste, selon Shier (1991, p. 92), à "garder les enfants heureux, occupés, hors de danger et loin des problèmes ». De plus, les malentendus sur ce qu'est et à quoi sert le playwork peuvent semer le doute et la confusion chez les professionnel-less quant aux interactions appropriées à avoir avec des enfants et peuvent engendrer des situations problématiques ou même dangereuses (Besse-Patin, Brougère \& Roucous, 2017). Être capable de définir la spécificité du playwork est ainsi devenue une préoccupation urgente et fondamentale du champ ces dernières années (Brown, Long \& Wragg, 2018).

Cet éternel problème du caractère propre au playwork a été rendu plus complexe du fait de ce que Hughes (2006) a nommé une "incursion» de professionnelle's d'autres milieux suite à l'introduction du référentiel de qualification du Playwork [National Occupational Standards for Playwork]. En effet, l'élaboration de ce référentiel a coïncidé avec la création et l'augmentation par le gouvernement de l'offre de prise en charge des enfants d'âge scolaire. Dès la fin des années 1980, des initiatives ont été prises pour financer et soutenir ces nouveaux accueils périscolaires (office for National Statistics, 1998 ; Smith \& Barker, 2000). Cette augmentation de l'offre de services de garde sur les temps périscolaires, le service de petit déjeuner à l'école ou l'ouverture de centres pendant les vacances, a entraîné une augmentation exponentielle du nombre de personnes pour lesquelles le playwork est devenu la seule qualification appropriée dans le cadre réglementaire nouvellement établi (Secrétariat d'État à l'éducation et à l'emploi et Secrétariat d'État à la sécurité sociale et Ministre des femmes, 1998). Alors 
qu'il n'existait pas de qualification pertinente, les adultes qui travaillaient avec des enfants dans ce large éventail de structures ont été poussés vers la formation et les qualifications du playwork, réglementées et financées par le gouvernement (Cordeaux et alii, 1999). Cette cohorte d'intervenant·e's « formée's ailleurs " (Russell, 1994, p. 1) a été accompagnée par de nouveaux et nouvelles formateur.rice's et évaluateur.rice's du playwork qui n'avaient pas ou peu de liens avec les origines du mouvement des terrains d'aventure. Ces derniers ont alors traduit et réinterprété ce que signifie «faire du playwork» à partir de leurs expériences et de leurs perspectives personnelles (Hughes, 2001). Avec ironie, Hughes (2006) remarquait alors qu'il y a eu probablement plus de personnes qui étudiaient le playwork que de personnes qui le pratiquaient effectivement.

Cette spécificité du playwork par rapport aux autres professions est souvent évoquée en parlant de "l'approche du playwork» (King \& Newstead, 2017). Entendue de façon générale comme "la façon "playwork" d'être, de faire, de penser et d'apprendre " (Handscomb \& Virdi, 2007, p. 17), l'expression reste (aussi) implicite et non définie (Conway, 2008). Au cours des quatre dernières décennies, une «succession d'examens tortueux de sa finalité et de la meilleure façon de le faire " (Hughes, 2012, p. 56) a été menée afin d'exprimer ce à quoi pourrait ressembler, en pratique, cette approche toujours indéfinie. Tout en produisant une littérature intéressante et stimulante en soi, ces réflexions ont conduit à diversifier considérablement les interprétations de ce qu'est le playwork et de ce à quoi il sert (Russell, 2012).

\section{Approvisionner et faciliter le « jeu libre»}

Pour les personnes travaillant dans le champ, la conception principale de ce que représente «faire du playwork» renvoie à l'idée de fournir des espaces et de faciliter ce que l'on appelle communément le "jeu libre", celui-ci étant compris comme un jeu sans programmation ni interférence des adultes. La raison d'être des terrains d'aventure en tant qu'espace de jeu a été développée dans les années 1970 et 1980, lorsqu'ils ont été menacés par un manque de financements et une nouvelle législation sur la santé et la sécurité (Hughes, 2006). En 1982, Hughes et Williams, playworkers du terrain d'aventure de Bristol, ont écrit une série d'articles sur l'importance du jeu pour les enfants dans le journal Play Times, une publication éditée par la NPFA. Le Comité National Mixte pour la Formation au Playwork [Joint National Committee on Training for Playwork] (JNCTP), un organisme s'occupant du développement des formations, a publié en 1985 des Recommandations sur la formation au playwork, également connues sous le nom de "livre saumon». Ce livre contenait la définition du jeu posée par Hughes, l'instituant comme le fondement du playwork, et qui est toujours utilisée aujourd'hui, sous une forme légèrement adaptée, dans les Principes du playwork (PPSG, 2005) : « Le jeu est un processus qui est librement choisi, dirigé personnellement et intrinsèquement motivé ». À partir de cette définition, Hughes a élaboré dans les années 1980 sa propre définition du playwork qui reste encore la plus utilisée aujourd'hui. Elle a été publiée pour la première fois par Playboard, un de ces organismes nationaux - éphémères - mis sur pied pour soutenir ce champ (Fewtrell, 1987) : « le playwork est l'acte spécifique de modifier "l'ensemble de l'environnement" avec l'intention délibérée d'améliorer les possibilités de jeu » (Playboard, 1984 cité dans Brown, 2003, p. 54) 
Dans ces espaces consacrés au jeu, le rôle de l'adulte est envisagé comme un pourvoyeur et un facilitateur du jeu (PPSG, 2005). Son rôle principal est la " modification de l'environnement " (King cité dans Hughes, 1996, p. 36) afin de rendre l'espace conforme à la forme de jeu décrite dans les Principes du playwork (2005). Pour ce faire, cela passe par l'organisation par les adultes d'«opportunités de jeu » (JNCTP, 1997 ; Stobart, 2001). On suppose régulièrement que les enfants tirent tout un éventail d'avantages à jouer dans ces espaces réaménagés pour le jeu, bien qu'il n'y ait que peu de preuves empiriques pour appuyer cette hypothèse. De plus, les playworkers se sont souvent demandés si les possibilités ainsi offertes ne limitaient pas en réalité le jeu des enfants, plutôt que de l'améliorer ou de l'enrichir (Williams, 1986). Malgré ces préoccupations, cette philosophie de ce qui est communément appelé « jeu libre » est devenue le fondement du champ du playwork britannique depuis quarante ans (Wilson, 2008b). Cependant, elle provoque plusieurs contradictions philosophiques et pratiques lorsqu'il s'agit de déterminer les spécificités voire la singularité de ces pratiques parmi les autres approches des métiers agissant auprès d'enfants. Conway (2003, p. 105) résume habilement ce problème de conceptualisation quand il est réduit à l'enrichissement de possibilités de jouer en déclarant que: «La pratique professionnelle du playwork est donc confrontée à la quadrature du cercle pour maintenir le sentiment d'autonomie de l'enfant et de contrôle sur ses propres expériences ludiques dans le cadre d'interventions d'adultes dans son espace et son temps de jeu».

Il y a une contradiction inhérente entre la notion de jeu revendiquée par le playwork et la conception du rôle de l'adulte qui en reste le pourvoyeur et le garant, en quelque sorte. Selon la perspective du playwork, le jeu est décrit comme un processus qui devrait être "librement choisi, dirigé personnellement et intrinsèquement motivé " par les enfants (PPSG, 2005). Littéralement, cela signifie que les enfants devraient décider de comment, avec quoi, et ce à quoi ils jouent; le «contenu et l'intention» du jeu devraient rester du ressort des enfants (Hughes, 1996). Or, concevoir le playwork en termes d'offre ou de facilitation légitime aux yeux de certains des pratiques adultes qui « retirent à l'enfant l'initiative et la conduite de l'action » (Bruner, 1986, p. 79). En tant que pourvoyeurs de jeu, les adultes s'adjugeraient la responsabilité de déterminer les «besoins de jeu» des enfants (Hughes, 1996) et d'y répondre en organisant et proposant des « opportunités de jeu " particulières (Meynell, 2008). Le jeu serait alors « enrichi par des playworkers qualifiés » (Play England, 2009) qui choisissent un «style d'intervention » qui prolonge le jeu (PPSG, 2005). En tant que facilitateurs du jeu, les adultes seraient placés en position d'autorité où, à la place des enfants, ils seraient responsables de la quantité et de la qualité des pratiques ludiques. Cette implication est perçue comme un risque d'une possible « adultérisation » (Else \& Sturrock, 2007, p. 93) $\mathrm{du}$ jeu des enfants. Traditionnellement décrite comme une pratique indésirable consistant à transformer le jeu des enfants suivant les idées et les programmes des adultes (Kilvington \& Wood, 2010), elle est d'ailleurs largement condamnée dans la pratique des autres professions (Morgan, 1999). En un sens, la conception actuelle du playworker comme un pourvoyeur et facilitateur de jeu est perçue comme légitimant l'introduction d'idées et de priorités émanant d'adultes dans les possibilités de jeu offertes aux enfants.

Par ailleurs, cette conception d'un adulte alimentant et facilitant le jeu conduit également à une vision dangereusement étroite de ce qui constitue une bonne pratique 
du playwork (Palmer, 2005). Au sein même du champ, on reconnaît depuis longtemps que le playwork est complexe et multidimensionnel (Abernethy, 1968). Pourtant les discussions de la littérature contemporaine sur le rôle des adultes se sont principalement concentrées sur ce qui est communément regroupé sous le terme d'« intervention » (Kilvington \& Wood, 2010). La description et la promotion d'une approche alternative du travail avec les enfants devraient permettre de définir ce que telle ou telle action proposée par les adultes engage "du point de vue du playwork», plutôt que de s'intéresser uniquement au jeu des enfants.

Ainsi la conception du playwork basée uniquement sur la définition d'un «jeu libre» limite la description des tâches des adultes à la seule dimension du jeu. Elle ne laisse aucune marge de manœuvre pour décrire ce que pourrait être une réponse selon «la perspective du playwork » à un large éventail d'événements - ludiques ou non - au sein de la vie quotidienne, par exemple, lorsque des enfants tuent des animaux ou parlent de sexualité. En conséquence, le champ du playwork a eu beaucoup de difficultés à déterminer les "réponses appropriées" à un ensemble de pratiques enfantines autogérées et à justifier ces réponses face à d'autres professions, notamment lorsqu'elles diffèrent de ce qui est courammeent considéré comme une bonne pratique (Lester, 2016).

\section{Les Principes du playwork}

Les Principes du playwork constituent les lignes directrices qui doivent inscrire la perspective de cette conception dans le travail avec les enfants, voire dans les Normes Professionnelles Nationales, en remplaçant d'anciens postulats (Bonel \& Lindon, 1996). Ils prétendent ainsi décrire en huit points, ce qu'il y a de singulier dans cette approche. Néanmoins, de nombreuses affirmations avancées dans les Principes sont des déclarations larges, dont des généralités sur l'importance du jeu pour les enfants qui sont partagées par d'autres professions et n'offrent donc rien de distinctif. De plus, la généralité des énoncés se prête facilement à l'interprétation et à l'application par d'autres professions et leurs paradigmes. Par exemple, "soutenir et faciliter le processus de jeu » peut être interprété dans de nombreuses perspectives personnelles et professionnelles. L'expression "prolonger leur jeu» sera interprétée par les playworkers comme «aider les enfants à jouer le plus possible hors des espaces prévus plutôt que de mettre un terme à ce jeu, simplement parce qu'il ne correspond pas aux attentes des adultes ». Or, l'expression « étendre leur jeu » est également utilisée dans le champ de la petite enfance où elle renvoie habituellement aux «stratégies utilisées pour soutenir et étoffer l'activité et les apprentissages qu'elle supporte " (Duncan \& Lockwood, 2008, p. 158). Ces deux interprétations qui peuvent être opposées conduisent à des conceptions complètement différentes du rôle de l'adulte en termes d'intervention dans le jeu des enfants. Elles créent des interprétations diverses de ce qui est requis au nom du playwork. En outre, les professionnelle-s peuvent difficilement comprendre ce que signifient en pratique des énoncés, tels que « le processus de jeu a la priorité ", dès lors qu'ils sont avancés sans explication dans les Principes du Playwork (Palmer, Wilson \& Battram, 2007).

Plutôt que de présenter la "perspective du playwork ", les Principes présupposent que les professionnelle-s la comprennent voire la devinent avant d'interpréter les points avancés. Cette absence de définition de l'approche du playwork n'a pas seulement 
conduit à des réinterprétations des pratiques du playwork, mais aussi à des transformations potentielles du travail lui-même. À la suite du remplacement des premiers postulats et valeurs du playwork par les Principes, Hughes (1997, p. 5) avait déjà noté : «J'ai visité de nombreuses organisations de playwork qui ont les valeurs affichées sur le mur du bureau et c'est généralement la dernière référence que j'ai formulée. La réalité est qu'ils annoncent les valeurs du Christ et mettent en œuvre les pratiques de Gengis Khan. »

Un problème supplémentaire des Principes réside dans leur définition du playwork et de sa visée principale : «créer ou fournir des espaces pour le jeu ». Ils ont repris sa conception spatiale renvoyant le rôle du playworker à celui de co-créateur d'espaces de jeu, plutôt que de décrire ce que signifie être un playworker dans ces espaces. Bien qu'il s'agisse d'un hommage à la pérennité du concept original des terrains d'aventure, cette vision était déjà dépassée avant même l'adoption du terme "playwork», comme nous l'avons vu précédemment. Malgré cela, cette définition physique et spatialisée est fermement ancrée dans l'imaginaire du playwork contemporain et se retrouve jusque dans les items des Normes Professionnelles Nationales du Playwork élaborées pour des institutions spécifiques comme les écoles, les prisons ou les hôpitaux (SkillsActive, 2012). De même, les polices d'assurance et les procédures de certification de la qualité reposent également sur cette même conception spatiale (Cambridge 2015). Dans ces "espaces de playwork», le rôle de l'adulte est pensé comme un pourvoyeur et un facilitateur du jeu (Newstead 2004), créant de fait plusieurs contradictions comme évoqué précédemment.

30 La récurrence dans la littérature de ce cette conception du playwork comme créateur d'espace de jeu pour les enfants tient à son intérêt et sa dimension universels. Mais c'est dans cette universalité que réside le problème fondamental d'une conception spatiale qui serait la caractéristique déterminante du playwork. En effet, mettre à disposition des opportunités ludiques aux enfants est une notion trop généraliste et généralisable, sans lien évident avec un ensemble de pratiques spécifiques adossées à des orientations philosophiques. Autrement dit, les enfants disposent aussi des possibilités de jeu dans les écoles, les crèches, les maisons, les musées, les parcs et les centres commerciaux, par l'entremise d'adultes dénommés éducateurs, parents, animateurs, architectes paysagistes ou gardiens d'enfants. En d'autres termes, il n'y a rien de « singulier » à offrir des espaces de jeu aux enfants.

31 Cette conception spatiale du playwork, devenue paradigmatique, s'inscrit en plus dans le domaine plus large de la promotion du jeu. Pourvoir et faciliter le jeu peuvent être interprétés selon des perspectives et des résultats très différents (Newstead, 2011), comme l'éducation, le sanitaire ou le développement social, visées qui ont toutes été rejetées par les playworkers (Williams, 1986; Morgan, 1999). Posé ainsi le playwork dans des termes généraux laisse à penser qu'il n'a rien de singulier ou de spécifique. Il devient légitimement un moyen d'insérer le jeu dans un cadre et des objectifs plus larges visant à améliorer l'apprentissage, le développement et le bien-être des enfants. Autant de perspectives pourtant critiquées par le playwork. Il se retrouve, de ce fait, englobé par des termes génériques et, incidemment, la littérature diffuse l'idée qu'il n'y a rien d'unique ou de spécifique au playwork. Paradoxalement, ce paradigme ouvre donc le playwork à toute une gamme d'interprétations contraires aux objectifs des pionniers des terrains d'aventure (Newstead, 2016). 


\section{Un service d'accueil}

Playcare est une autre appellation choisie pour décrire le service fourni par le nouveau gouvernement qui développa des accueils périscolaires dans les années 1990 (Davy, 1995). Basée sur une combinaison du jeu et du soin, cette pratique a été le thème de plusieurs ouvrages qui s'adressaient directement à celles et ceux que l'on appelait des playworkers et travaillaient dans des structures d'accueil où les enfants étaient placés du fait du besoin de garde des parents et ne pouvaient pas être pris en charge par l'école. Ce service était très différent de l'idée généralement admise du playwork reposant sur les «trois libertés » (Else, 2009) : liberté d'accès, liberté de circulation, et liberté de faire. Mais l'absence d'une définition formelle quelconque du playwork qui aurait permis de le raccrocher à ses origines largement extérieures (plein air), utilisant des matériaux recyclés et sous l'égide des enfants, a conduit à ce que celui-ci soit (ré)interprété une fois de plus par et pour ce nouveau groupe professionnel. Il a pris alors une forme opposée avec des espaces souvent intérieurs ainsi que des activités élaborées et programmées par des adultes. Bien que plusieurs playworkers ayant d'abord travaillé dans les terrains d'aventure aient participé à l'élaboration de ces ouvrages, la réinterprétation du playwork dans le cadre de la garde d'enfants a pu scandaliser de nombreuses personnes. À travers d'autres publications, elles ont marqué leur opposition principalement sur les aspects d'organisation des activités ludiques et du temps des enfants dans ces structures d'accueil. Pendant que les pionniers des terrains d'aventure luttaient encore pour définir le playwork, beaucoup d'entre eux étaient néanmoins fermement convaincus que, quoi que ce soit, ce n'était pas de la "garde d'enfant ». Et certains d'entre eux sont allés jusqu'à affirmer que cette réincarnation scolaire ne devrait surtout pas être associée au playwork (Milne, 1998).

\section{Un playwork évolutionniste}

L'expression de "playwork évolutionniste » est avancée par Hughes en titre de son ouvrage de référence publié pour la première fois en 2001, Playwork évolutionniste et la pratique de l'analyse réflexive. Appuyé sur la psychologie évolutionniste et du développement, sa théorisation du playwork est basée sur la notion de "privation du jeu ». En arguant pour une approche évolutive, il plaide en faveur de la mobilisation d'espaces qui peuvent compenser ce qu'il croit être une diminution - voire la privation - du jeu dans la vie des enfants. Le jeu étant pour lui essentiel à la survie de notre espèce, Hughes craint que sa privation n'ait un effet important sur le bien-être actuel et le développement futur des enfants. Par conséquent, il considère que le rôle du playworker est de fournir un " antidote » aux tentatives de la société moderne d'écarter le jeu de la vie des enfants.

Comme dans tous ses écrits, l'œuvre majeure de Hughes (2001) présente de nombreux exemples et suggestions pratiques tirés de sa longue carrière professionnelle dans le playwork. Cependant, les anecdotes personnelles et les études de cas ne parviennent pas à fournir une description systématique de cette approche qui pourrait être reproduite par d'autres. Malgré les nombreux exemples de ce qui serait admis comme des « bonnes pratiques ", la conception évolutionniste demeure peut-être davantage un concept ou une finalité du playwork qu'une analyse claire de ce qu'il est et comment il diffère des autres approches du travail avec les enfants. Par ailleurs l'approche évolutionniste de 
Hughes conduit à interroger la réalité empirique de la privation du jeu chez les enfants. Et si elle existe, dans quelle mesure le jeu est-il amélioré par le playwork plutôt que réduit? Les pionniers des terrains d'aventure ont très clairement affirmé que leur travail de gestion de terrains vagues n'était qu'un «supplément » (Abernethy, 1968) et non une panacée. Comme le disait Benjamin (1974, p. 3) : « Notre problème n'est pas de concevoir des rues, des logements, une station-service ou des magasins qui peuvent se prêter au jeu, mais d'éduquer la société à accepter les enfants et leur participation ». Pour eux, le rôle des terrains d'aventure n'était qu'une solution temporaire à un problème social beaucoup plus vaste que le jeu en tant quel, alors que pour Hughes, le (manque de) jeu est le problème et le playwork la solution.

\section{Un playwork thérapeutique}

Else \& Sturrock (2007) ont avancé la notion de "playwork thérapeutique ». Leur idée, selon laquelle les playworkers pouvaient aider les enfants à accéder au potentiel créatif et curatif du jeu, a été reprise dans tout le Royaume-Uni et à l'échelle internationale, dans le cadre de projets d'intervention auprès d'enfants jusqu'à des programmes universitaires. Diverses interprétations coexistent, allant du travail avec des enfants ayant un comportement difficile dans des accueils périscolaires ordinaires à des interventions sur mesure pour les enfants traumatisés ou vulnérables (Hawkes, 2017; Webb \& Brown, 2003). Quel que soit le contexte, la compréhension des bienfaits thérapeutiques du jeu et de la façon dont certaines actions et réactions des adultes les améliorent (ou diminuent) potentiellement pourrait simplement être considérée comme une connaissance et une compétence supplémentaires du playworker. Cependant, le fait d'utiliser spécifiquement le jeu pour aider les enfants et obtenir des bienfaits thérapeutiques pourrait aussi entrer en contradiction avec le dicton généralement accepté du playwork, à savoir : « faible intervention [des adultes], réponse élevée [des enfants]» (Hughes, 1996, p. 51). Autrement dit, les enfants sont censés pouvoir intervenir sur les formes d'attention qu'ils reçoivent de la part des adultes. Pour citer encore Hughes (1996, p. 51), «pas de méthode, pas de besoins ». Le recours au jeu pour obtenir des bienfaits thérapeutiques, même s'il est utile pour des enfants, suggère l'imposition d'une organisation adulte du jeu des enfants et pourrait donc être considérée comme une forme d'adulterisation (Else \& Sturrock, 2007), selon la perspective du playwork.

\section{Le playwork comme pratique}

Depuis les débuts des terrains d'aventure britanniques, le playwork a été mobilisé dans une grande variété d'espaces et de structures : des hôpitaux aux prisons, des maisons aux parcs, etc. Ces dernières années, on s'est intéressé de plus en plus à la conception du playwork comme une forme de pratique qui ne se limite pas à des structures identifiées sous cette appellation. Plusieurs auteur'e's ont ainsi plaidé en faveur de la nécessité de "sortir de l'hibernation " (Gladwin, 2008) en concevant le playwork comme une pratique sans frontières tout en s'assurant que ce qu'il signifie est adaptable à tout espace ou structure (Kilvington, Knight \& Sexton, 2008 ; Kingston, 2008). Le lancement de l'International Journal of Playwork Practice en 2014 a mis en évidence cette approche non spatiale du playwork à l'échelle mondiale. 
L'expression de "pratique de playwork» est fréquemment utilisée dans la littérature contemporaine. Elle renvoie le plus souvent à ce qui est fait pour «faire du playwork » ou une manière de faire les choses selon la perspective du playwork (Play England, 2008). Pour autant, la pratique est toujours peu définie dans la littérature. La définition qui figure dans les Normes Professionnelles Nationales du Playwork stipule que la pratique est «ce que vous faites dans l'environnement pour mettre en œuvre les Principes du playwork, y compris ce que vous dites et ne dites pas et ce que vous faites et ne faites pas » (SkillsActive, 2010, p. 2). Toutefois, cette définition est problématique à deux égards. D'une part, les Principes du playwork ne décrivent pas comment le mettre en pratique (Brown, 2008). D'autre part, la référence implicite à ce qui n'est pas " effectué » au nom du playwork pose un défi existentiel pour les playworkers. Pour les initiés qui interprètent cette déclaration, de l'intérieur, le sens est clair : ce qui n'est "pas dit» et "pas réalisé » au nom du playwork, ce sont les actions que les autres professions sont censées faire en termes de direction et d'organisation des activités. Prise de façon littérale, articuler le playwork en termes de ce qui n'est pas dit et de ce qui n'est pas fait devrait, en toute logique, inclure de façon paradoxale tout ce que le playwork n'est pas.

$\mathrm{Au}$ cours des dernières années, on constate un intérêt croissant pour la perspective du Playwork Action Research System (PARS) développé dans la thèse de Newstead (2016) et qui est maintenant utilisée dans plusieurs pays à travers le monde. Le modèle du PARS repose sur une conception du playwork, non pas en termes d'espace ou de mode d'action spatialisé, mais en tant que méthodologie spécifique de travail avec les enfants qui peut être utilisée par toutes les professions. Son objectif est de permettre aux adultes qui interviennent dans les différentes structures de décrire et d'analyser leur pratique à partir de la perspective du playwork. Cette approche consiste à structurer les pratiques en s'appuyant sur les interventions actions et les interactions des adultes avec les enfants plutôt qu'à partir de considérations uniquement matérielles et spatiales. Selon plusieurs travaux, cette méthodologie a un effet significatif sur les pratiques des adultes qui travaillent avec les enfants dans des métiers à la marge du playwork, comme les enseignants de maternelle (Chan, sous presse).

\section{Encore un long chemin...}

Malgré des décennies de débats - souvent passionnés -, la profession du playwork n'est pas plus avancée pour formuler une définition qui pourrait décrire sa spécificité qu'elle ne l'était aux premiers jours des terrains d'aventure. Au cours des quarante dernières années, de nombreuses personnes ont consacré beaucoup d'efforts à démontrer la valeur de leur travail en focalisant sur la valeur au jeu. Bien que ce "mantra ludique » (Candler, 1999, p. 230) soit profondément ancré dans l'imaginaire du playwork, il n'a pas réussi à constituer l'identité d'une approche singulière du travail avec les enfants (Lester, 2016). Ces dernières années, des terrains d'aventure et plusieurs projets de playwork à travers le Royaume-Uni ont été fermés en raison d'une combinaison des mesures d'austérité du gouvernement britannique et de l'incapacité à justifier leur propre existence de façon convaincante. De plus, le métier de playworker s'est homogénéisé avec d'autres métiers agissant auprès d'enfants, conséquence de ses difficultés à mettre en avant sa spécificité (Podyma, 2015). Encore récemment, le 
playwork est souvent présenté sous un faux jour renvoyant, par exemple, à la garde d'enfants ou à la prévention du crime (Wragg, 2008).

Cette question d'identité est peut-être encore plus importante pour la survie et le développement du playwork et sa crédibilité comme alternative à d'autres métiers en relation avec les enfants. De nos jours, le terme de playwork est utilisé pour décrire un large éventail de métiers et d'institutions à travers le monde (Wong, 2015). Cependant, comme le fait remarquer Sutton (2014), si cet intérêt international est apparemment une évolution positive, il comporte aussi des risques inhérents pour la reconnaissance future de sa pertinence. Or, actuellement, il n'y a pas de recherche sur la façon dont le playwork est compris dans le monde. Au vu des difficultés britanniques depuis sa création, il est possible (et vraisemblable) qu'il y ait autant de définitions et d'interprétations supplémentaires qu'il y ait de personnes qui le mettent en pratique dans d'autres pays.

41 En l'absence d'une identité stable et repérée qui le distingue, le playwork risque d'être réduit ou anéanti par d'autres professions de l'enfance mieux établies et reconnues (Stobart, 2008). Ce que l'on peut (et devrait) retenir de l'histoire du playwork est qu'un manque de clarté sur la nature et les finalités d'un métier conduit à sa fragmentation et à la prolifération d'autres modalités de pratique au nom de mêmes principes, voire sous le même nom. En l'absence de productions ou de formations de la part des pionniers qui expliquent de façon cohérente la nature et le but des terrains d'aventure, ces derniers ont été orientés et utilisés différemment. Un rapport de la NPFA (1960, p. 3) note que les terrains d'aventure "se sont développés dans des directions différentes sous l'influence des playleaders... ", et Benjamin (1961, p. 7) se plaint que certains terrains d'aventure « adoptent la forme de programme organisé qui avait la préférence dans les années $30 »$. Autre exemple, Spencer, Tuxford \& Dennis (1964) ont documenté la diversité des visions au sein d'un même comité de gestion d'un terrain d'aventure à Bristol. Dans les années 1970, certains terrains servaient de moyens pour répondre " aux besoins des enfants, des adolescents délinquants, des mères et des retraités, en fait de n'importe quelle personne qui n'est pas accueillie » dans les autres institutions (Burkhardt, 1977, p. 147). De même, dans les années 1990, lorsque le playwork est devenu la «qualification à la mode » pour quiconque travaillait avec des enfants (hors des professions établies), l'absence de définition du playwork signifiait que ceux qui arrivaient sur le terrain avec une variété d'expériences professionnelles étaient libres de le traduire à leur façon. Et, pour de nombreux auteurs, les pratiques réalisées au nom du playwork allaient à l'encontre des valeurs qui avaient présidé au lancement de ce métier.

Avec l'intérêt international croissant pour le playwork, l'histoire semble actuellement se répéter, mais pas nécessairement en favorisant son développement. Les personnes qui adoptent le terme playwork en dehors du Royaume-Uni peuvent aussi provenir de milieux professionnels et de philosophies très divers, et utiliser simplement les éléments de la théorie ou des Principes du playwork qui correspondent le mieux à leurs intentions pour justifier leur forme particulière de pratiques. Dans de nombreux pays à travers le monde, il n'existe que peu ou pas de formation ou de qualifications professionnelles dans ce domaine. Même si de tels développements ont eu lieu, sans une définition communément admise du playwork et des façons de le mettre en pratique au quotidien, il n'y a aucune garantie que la formation ou les qualifications contribuent à une meilleure compréhension de l'approche. Par ailleurs, il est probable que ceux qui 
soutiennent le développement professionnel du champ du playwork aient besoin de s'appuyer sur leur propre expérience et leur propre compréhension, concourant ainsi à développer davantage de variations sur le playwork.

\section{Conclusion}

Cet article a exploré le développement historique de l'identité du playwork au cours des soixante-dix dernières années en s'appuyant sur la littérature qui lui a été consacrée. Les analyses montrent que pour devenir une profession établie au RoyaumeUni et proposer une approche alternative à d'autres métiers intervenant auprès d'enfants à l'échelle mondiale, il reste à résoudre des questions déjà anciennes sur la nature et les visées du playwork. Il s'agirait de s'interroger sur la valeur du «mantra ludique » cher aux playworkers et de se demander si, contrairement à la focalisation du débat pendant ces quarante dernières années, le développement de la profession ne serait pas mieux servi en se concentrant sur la nature et le but du playwork, plutôt que sur la nature et le but du jeu. Jusqu'à ce qu'il y ait un accord universel sur la nature et l'objectif de ce que Else (1997, p. 42) réfère au "véritable playwork", celui-ci sera adapté et adopté pour répondre aux besoins de tout dispositif et toute tendance politique. Car, comme l'a observé Heseltine (1982) dans sa conférence : « Nous devons savoir non seulement ce que nous voulons réaliser, mais aussi pourquoi nous voulons le faire. Cela permettrait aux playworkers sur le terrain de savoir pourquoi ils travaillent et vers quel but... Il se peut que nous parlions de changements fondamentaux dans notre société et de la façon dont elle voit ses enfants. Le jeu et l'encadrement du jeu [leadership] ne sont que les moyens d'atteindre une fin, mais nous en sommes venus à les considérer comme la fin. Probablement parce qu'on ne sait pas encore quelle est la vraie fin.»

\section{BIBLIOGRAPHIE}

ABERNETHY W. D. (1968), Playleadership, London, National Playing Fields Association.

ABERNETHY W. D. (1984), « History of Playwork », in Playwork : Bases, Methods and Objectives conference, PlayEducation.

ALLEN OF HURTWOOD M. (1946), « Why Not Use Our Bomb Sites Like This ? », Picture Post, 16th November 1946, pp. 26-27.

ALLEN OF HURTWOOD M. (1953), Proposed Organisation for Initiating Adventure Playgrounds, University of Warwick, Modern Records Centre.

ALLEN OF HURTWOOD M. (1954), « Praise for Emdrup and possibility of writing a book », Personnal letter to J. BERTELSEN, University of Warwick, Modern Records Centre.

ALLEN OF HURTWOOD M. (1961), Adventure playgrounds, London, National Playing Fields Association. 
ALLEN OF HURTWOOD M. (1964), New playgrounds, London, The Housing Centre Trust.

ALLEN OF HURTWOOD M. \& NICHOLSON M. (1975), Lady Allen of Hurtwood Memoirs of an Uneducated Lady, London, Thames and Hudson.

ANDERSSON S.-I. \& HøYER S. (2001), C. Th. Sørensen : Landscape Modernist, Copenhagen, Danish Architectural Press.

ARMITAGE M. (2014), « Playwork : the anarchy wing of sociology », in C. BURKE \& K. JONES (eds.), Education, Childhood and Anarchism : Talking Colin Ward, Abingdon, Routledge.

BALMFORTH N. (1982), « Looking At Play », Play Times, 31-35.

BANKS S. (2010), Ethical Issues in Youth Work, Abingdon, Routledge.

BARRAND R. G. (1972), Forming an Adventure Playground, Parks and Sports Grounds.

BENGTSSON A. (1970), Environmental Planning for Children's Play, New York, Praeger.

BENGTSSON A. (1975), « Adventure Playgrounds and Children's Creativity », in IPA 6th International Conference, University Bucconi.

BENJAMIN J. (1961), In Search of Adventure : A Study of the Junk Playground, Leicester, Blackfriars Press.

BENJAMIN J. (1974), Grounds for Play, London, National Council of Social Service.

BESSE-PATIN B., BROUGÈRE, G. \& ROUCOUS, N. (2017), « Losing the "Monopoly" : A French Experience of Playwork Practice ", Journal of Playwork Practice, 4, 1, pp. 23-37.

BONEL P. \& LINDON J. (1996), Good Practice in Playwork, Cheltenham, Stanley Thornes.

BROWN F. (ed.) (2003), Playwork: Theory and Practice, Buckingham, Open University Press.

BROWN F. (2008), « The Playwork Principles : a Critique », in F. BROWN \& C. TAYLOR (eds.), Foundations of Playwork, Maidenhead, Open University Press, pp. 123-127.

BROWN F., LONG A. \& WRAGG M. (2018), « Playwork : A Unique Way of Working with Children », in P. K SMITH. \& J. L. ROOPNARINE (eds.), The Cambridge Handbook of Play, Cambridge, Cambridge University Press, pp. 704-721.

BRUNER J. S. (1986), « Play, thought and language », Prospects - Quarterly Review of Education, XVI, pp. 77-83.

BURKHARDT B. (1977), The Educational Function and Facilities of Adventure Playgrounds, London, University of London.

CANDLER P. A. (1999), Cross-national Perspectives on the Principles and Practice of Children's Play Provision, Leicester, De Montfort University.

CHAMBERS'S JOURNAL (1884), « Over-Educating Children ». Chambers's Journal, $7^{\text {th }}$ June, pp. 366-367.

CHAN P. (forthcoming), « Playwork Play Works », in J. CARTMEL \& W. RICK (eds.) Playwork at the Margins, Abingdon, Routledge.

CHARMAZ K. (2003), « Grounded Theory : Objectivist and Constructivist Methods », in N. K. DENZIN \& Y. S. LINCOLN (eds.), Strategies of Qualitative Inquiry, Thousand Oaks, Sage.

CHILTON T. (2013), Adventure Playgrounds - a brief history, Bognor Regis, Fair Play for Children. CENTRAL COUNCIL OF RECREATIVE PHYSICAL TRAINING (1937), Play Leadership : Its Development and Organization, London, CCRPT \& NPFA. 
CONWAY M. (2003), « Professional Playwork Practice », in F. BROWN (ed.), Playwork - Theory and Practice, Maidenhead, Open University Press.

CONWAY M. (2008), « The Playwork Principles », in F. BROWN \& C. TAYLOR (eds.), Foundations of Playwork, Maidenhead, Open University Press, pp. 119-122.

CORDEAUX C., HALL B., OWEN S. \& MILES R. (1999), Child Care Training in the United Kingdom : Final report, Ipswich, Suffolk County Council Social Services.

CROWTHER S. (1968), Play in London, Report, London, London Adventure Playground Association.

CURTIS H. S. (1915), The Practical Conduct of Play, New York, Macmillan.

DALLAL J. (2015), « A quest to professionalise playwork through higher education », Journal of Playwork Practice, 2, pp. 71-75.

DAVY A. (1995), Playwork: Play and Care for Children 5-15, Andover, Macmillan.

DAVY A. (2007), « Playwork : Art, Science, Political Movement or Religion ? », in W. RUSSELL, B. HANDSCOMB \& J. FITZPATRICK (eds.), Playwork Voices : In Celebration of Bob Hughes and Gordon Sturrock, London, London Centre for Playwork Education and Training.

DUNCAN J. \& LOCKWOOD M. (2008), Learning Through Play: A Work-Based Approach for the Early Years Professional, New York, Continuum.

DYKE M. (1997), « Reflective learning as reflexive education in a risk society : empowerment and control ? », International Journal of Lifelong Education, 16, pp. 2-17.

ELSE P. (1997), « Managing Personal Behaviour - a View of Reflective Playwork », in P. ELSE \& G. STURROCK (eds.), Therapeutic Playwork Reader one 1995-2000, Eastleigh, Common Threads.

ELSE P. (2007), An Ethical Statement for Playworkers, London, Joint National Committee on Training for Playwork.

ELSE P. (2009), The Value of Play, London, Continuum International.

ELSE, P. \& STURROCK, G. (2007), Therapeutic Playwork Reader one 1995-2000, Eastleigh, Common Threads.

FEWTRELL J. (1987), « PlayBoard Liquidation », Schools Out, London, Kids Clubs Network.

FROST J. L. (2012), « The changing culture of play », International Journal of Play, 1, pp. 117-130.

GARVEY D. \& LANCASTER A. (2010), Leadership for Quality in Early Years and Playwork : Supporting your Team to Achieve Better Outcomes for Children and Families, London, National Children's Bureau.

GEARY L. (1972), A Play Leadership Handbook, Nottingham, Nottinghamshire Playing Fields Association.

GLADWIN M. (2008), « Let's get off our ice floe and into the swim : a provocation ", in Possible Futures for Playwork, London, Play England.

HANDSCOMB B. \& VIRDI M. (2007), « Playwork Learning - Sharing the Journey », in W. RUSSELL, B. HANDSCOMB \& J. FITZPATRICK (eds.), Playwork Voices : In Celebration of Bob Hughes and Gordon Sturrock, London, London Centre for Playwork Education and Training.

HAWKES C. (2017), « Therapeutic playwork : exploring playworkers' perceptions of therapeutic playwork training and its usefulness in supporting children in afterschool clubs ", in W. L. RUSSELL \& H. STUART SMITH (eds.), Practice-based Research in Children's Play, Bristol, Policy Press. HESELTINE P. (1982), « Review of the current state of play », in The Transcript conference, Bolton, PlayEducation. 
HOLME A. \& MASSIE P. (1970), Children's Play : A study of Needs and Opportunities, London, Michael Joseph Publishing.

HUGHES B. (1996), Play Environments : A Question of Quality, London, PLAYLINK.

HUGHES B. (1997), « Towards a technology of playwork », in From Values to Practice - Rethinking Playwork Training, London, PLAYLINK.

HUGHES B. (2001), Evolutionary Playwork and Reflective Analytic Practice, London, Routledge.

HUGHES B. (2006), Play Types Speculations and Possibilities, London, London Centre for Playwork Education and Training.

HUGHES B. (2012), Evolutionary Playwork and Reflective Analytic Practice, 2nd edition, London, Routledge.

INTERNATIONAL PLAYGROUNDS ASSOCIATION (1975), « Adventure Playgrounds and Children's Creativity », IPA 6th International Conference, University Bucconi.

JAGO L. (1972), IPA Conference at Vienna, London, London Adventure Playground Association.

JOINT NATIONAL COMMITTEE ON TRAINING FOR PLAYWORK (1997), What's A Playworker Then?, London, JNCTP.

JOINT NATIONAL COMMITTEE ON TRAINING FOR PLAYWORK (1985), Recommendations on Training for Playwork (The Salmon Book), London, JNCTP.

KILVINGTON J., KNIGHT H. \& SEXTON J. (2008), « Playwork : a profession or 'poor relation' ? Ideas Paper 10 », in Possible Futures for Playwork. London, Play England.

KILVINGTON J. \& WOOD A. (2010), Reflective Playwork : For All Who Work with Children, London, Continuum International.

KING F. (1988), « Playwork - The Challenge Pt 2 », in PlayEd 1987 - 1988 Part 2 conference. PlayEducation.

KING P. \& NEWSTEAD S. (Eds.) (2017), Researching Play from a Playwork Perspective, Abingdon, Routledge.

KINGSTON B. (2008), « Playwork - Mostly harmless? », in Possible Futures for Playwork, London, Play England.

LAMBERT J. (1974), Adventure Playgrounds : A Personal Account of a Play-leader's Work, as told to Jenny Pearson, London, Penguin Books.

LAVE J. \& WENGER E. (1991), Situated Learning : Legitimate Peripheral Participation, Cambridge, Cambridge University Press.

LESTER S. (2016), The Value of Playwork Provision, Manchester Manchester, Playwork Northwest.

LEICHTER SAXBY, M. \& LAW S. (2015), The New Adventure Playground Movement : How Communities across the USA are Returning Risk and Freedom to Childhood, Notebook Publishing.

MACLEAN M., RUSSEL W. \& RYALL E. (eds.) (2015), Philosophical Perspectives on Play, London, Routledge.

MAYS J. B. (1957), Adventure in Play, Liverpool, Liverpool Council of Social Service.

McKENDRICK J. H., KRAFTL P., MILLS S., GREGORIUS S. \& SYKES G. (2015), « Complex geographies of play provision dis/investment across the UK », International Journal of Play, 4, pp. 228-235. 
MEYNELL W. (2008), « Thinking about Creating a Play Environment », in F. BROWN \& C. TAYLOR (eds.), Foundations of Playwork, Maidenhead, Open University Press, pp. 113-115.

MILNE J. (1998), «Conflict of Terminology - definitions of playwork and childcare », in Playwords, Eastleigh, Common Threads.

MILNE J. (2014), « An Adventure Playground for Halabja », Journal of Playwork Practice, 1, pp. 229-231.

MORGAN B. (1999), « Is some 'Playwork’ Damaging Children? », in Playwords, Eastleigh, Common Threads.

MORTENSON D. (2014), « Imagine. Create. Build. An adventure in the forest », Journal of Playwork Practice, 1, 2, pp. 209-210.

NATIONAL CHILDREN'S PLAY AND RECREATION UNIT (1991), Accrediting Playwork(ers) - a scheme for accrediting the competences of play workers, London, NCPRU.

NATIONAL PLAYING FIELDS ASSOCIATION (1957), Report of a conference on play leadership on adventure playgrounds, London, NPFA.

NATIONAL PLAYING FIELDS ASSOCIATION (1960), Adventure Playgrounds - a progress report, London, NPFA.

NEWSTEAD S. (2004), The Buskers Guide to Playwork, Eastleigh, Common Threads.

NEWSTEAD S. (2011), The Buskers Guide to Playwork, 2nd edition, Eastleigh, Common Threads.

NEWSTEAD S. (2016), De-constructing and Reconstructing the Unorthodox Recipe of Playwork, Doctoral thesis, University College London.

NICHOLLS D. (2002), Employment practice and policies in youth, community and play work: in pursuit of fairness at work, Lyme Regis, Russell House.

NICHOLSON M. (1954), Notes on Adventure Playgrounds, London, National Playing Fields Association.

OFFICE FOR NATIONAL STATISTICS (1998), Out of School Childcare Initiative : An Evaluation of LongTerm Sustainability. In DFEA (ed.), Employment, London, HMSO.

PALMER S. (2005), « Does Quality Training and Education ensure Quality Playwork? », in H. HUNTER \& J. MARTIN (eds.), Playwork Qualifications Raising the Standard. A Report of the JNCTP, London, JNCTP.

PALMER M., WILSON P. \& BATTRAM A. (2007), « The playing that runs through us all : illustrating the Playwork Principles with stories of play », in W. RUSSELL, B. HANDSCOMB \& J. FITZPATRICK (eds.), Playwork Voices : In Celebration of Bob Hughes and Gordon Sturrock, London, London Centre for Playwork Education and Training, pp. 121-138.

PLAYEDUCATION (1987) PlayEd 1987 - 1988 Part I, London, PlayEducation.

PLAY ENGLAND (2008), Quality in Play, London, Play England.

PLAY ENGLAND (2009), Quality in Play, London, Play England.

PLAYLINK (1997), From Values to Practice - Rethinking Playwork Training, London, Playlink.

PLAYWORK PRINCIPLES SCRUTINY GROUP (2005), The Playwork Principles, London.

PODYMA R. (2015), « The efficiency of playwork as a universal service », Journal of Playwork

Practice, 2. 
RAMER S. L. (2005), « Site-ation pearl growing : methods and librarianship history and theory », Journal of Medical Library Association, 93, 3, pp. 397-400.

ROBERTS B. (2002), Biographical research, Buckingham, Open University Press.

ROSATI L. S. (1975), « The Community, The Law and Adventure Playgrounds », in IPA 6th International Conference, University Bucconi.

RUSSELL W. (1994), « JNCTP and ? in the Nineties. Welcome speech by Wendy Russell », in JNCTP AGM 1994 Coming of Age, London, JNCTP.

RUSSELL W. (2005), « Slices of history from the JNCTP fruit cake from childhood (1990) to adolescence (2005) » in Celebrating a Safe Space for Dialogue: The History and Future of Playwork Training. 30th Birthday Party Conference, Birmingham, JNCTP.

RUSSELL W. (2012) « "I get such a feeling out of... those moments" : playwork, passion, politics and space », International Journal of Play, 1, pp. 51-63.

SECRETARY OF STATE FOR EDUCATION AND EMPLOYMENT \& THE SECRETARY OF STATE FOR SOCIAL SECURITY AND MINISTER FOR WOMEN (1998), The Green Paper - Meeting The Childcare Challenge - A Framework And Consultation Document, Sudbury, DfEE Publications.

SHIER H. (1990), Playwork Accreditation Report. London, JNCTP.

SHIER H. (1991), « Exploring logo in children's play : A playworker's perspective », Early Child Development and Care, 69, 1, pp. 91-97.

SHIER H. (1996), « Time for playworkers to unionise », Report, Birmingham, CYWU.

SKILLSACTIVE (2006), Quality Training, Quality Play 2006-2011 : The First UK Strategy for Playwork Education, London, SkillsActive.

SKILLSACTIVE (2010), PW30 Carry out playwork in a school setting, London, SkillsActive.

SKILLSACTIVE (2012), Review of New Additional Playwork National Occupational Standards, London, SkillsActive.

SMITH F. \& BARKER J. (2000), « Contested Spaces Children's Experiences of Out of School Care in England and Wales », Childhood, 7, 3, pp. 315-333.

SøRENSEN C. T. (1931), Parkpolitik i Sogn og Købstad, København, Kommission hos gyldendalske boghandel nordisk forlag.

SøRENSEN, C. T. (1947) « Skrammellegeplads », in M. ALLEN OF HURTWOOD (ed.), Modern Records Centre, University of Warwick.

SPENCER J. C., TUXFORD J. \& DENNIS N. (1964), Stress and Release in an Urban Estate : a Study in Action Research, London, Tavistock Publications.

STOBART T. (2001), Take Ten for Play Portfolio, Brixham, Furzeham Graphics.

STOBART T. (2008), « Playwork as a profession : distinct or extinct? », in F. BROWN \& C. TAYLOR (eds.), Foundations of Playwork, Maidenhead, Open University Press, pp. 18-21.

STONEHOUSE D. (2015), « Ethical practice for the playwork practitioner », International Journal of Play, 4, pp. 314-323.

SUTTON L. (2014), « Adventure playgrounds and environmental modification : a beginner's guide ", Journal of Playwork Practice, 1, 2, pp. 211-217.

THE CRAWLEY COMMUNITY ASSOCIATION ADVENTURE PLAYGROUND COMMITTEE (1955), Crawley Adventure Playground, Crawley, TCCAAPC. 
THE RURAL DISTRICT COUNCIL OF WELLINGTON (1963), An Experiment in Play Leadership, Shropshire, The Rural District Council of Wellington.

TOSH J. \& LANG S. (2006), The Pursuit of History: Aims, Methods and New Directions in the Study of Modern History, Harlow, Pearson Longman.

TURNER H. S. (1961), Something Extraordinary, London, Michael Joseph.

VAN ROOIJEN M. \& NEWSTEAD S. (2016), « Influencing factors on professional attitudes towards risk-taking in children's play : a narrative review », Early Child Development and Care, 187, 5-6, pp. 946-957.

WATERS P. (2014), « Into the Woods : Stories and Nature in Playwork Training », Children, Youth and Environments, 24, 3, pp. 221-235.

WEBB S. \& BROWN F. (2003), « Playwork in Adversity : working with abandoned children in Romania », in F. BROWN (ed.), Playwork Theory and Practice, Maidenhead, Open University Press, pp. 157-175.

WILLIAMS H. (1986), Playworks, Lancaster, PlayEducation.

WILSON P. (2008a), « Passion, recalcitrance, sound management and confident applications of the craft », Possible Futures for Playwork. London, Play England.

WILSON P. (2008b), « The Cultural Origins and Play Philosophy of Playworkers : An Interview with Penny Wilson », American Journal of Play, 1, pp. 269-282.

WONG A. (2015), « Key themes from playwork training in Hong Kong », Journal of Playwork Practice, 2,1, pp. 85-87.

WRAGG M. (2008), « Guerilla Playwork », in F. BROWN \& C. TAYLOR (eds.), Foundations of Playwork, Maidenhead, Open University Press, pp. 169-173.

WRAGG M. (2016), « Play and Playwork in the Prison : past, present and future », Prison Service Journal, 223, pp. 5-10.

\section{NOTES}

1. NdT : Nous tenons à remercier Marc Carletti pour l'aide apportée lors de la traduction. Comme indiqué dans l'introduction au dossier, le terme orignal de "playwork» a été conservé car sa signification comme les pratiques qu'il représente ne sauraient se confondre avec ce que recouvre le terme " animation » en français ni avec d'autres pratiques professionnelles basées sur le jeu.

2. 'NdT : On peut noter le titre quelque peu provocateur de cet article : Pourquoi ne pas utiliser nos sites bombardés ainsi ?. Pour l'anecdote, activiste pour le soin et les droits des enfants - dont a pu résulter le Children Act en 1948 - et des femmes, l'auteure a investi deux premiers sites bombardés avant d'en installer un troisième à Lollard, devant le parlement britannique et ainsi forcer les députés à constater sa réussite.

3. $\mathrm{NdT}$ : Les "playschemes" sont habituellement des programmes d'activités et de jeux mis en place ponctuellement durant les vacances, notamment estivales, en investissant des lieux comme les cours d'école.

4. $\mathrm{NdT}$ : Contrairement aux playschmes, les playcentres fonctionnaient de façon permanente et s'établissaient dans des bâtiments dédiés pour mettre en place le même type de programmes durant l'année en fonction des besoins de la collectivité. Ils s'apparentaient, en ce sens, aux centres de loisirs en France. 


\section{RÉSUMÉS}

Le playwork est une approche alternative pour travailler avec les enfants qui a été développée par les pionniers des terrains d'aventure au Royaume-Uni après la Seconde Guerre mondiale. Malgré une histoire riche et l'existence d'un statut professionnel de playworker au Royaume-Uni, nous savons peu de choses sur la façon dont cette pratique s'est constituée depuis son origine. Cet article décrit l'évolution historique de ce métier qui est passé d'une communauté de pratiques jusqu'à une profession reconnue nationalement et qui se développe maintenant en un mouvement international. Il souligne ainsi comment le playwork a été défini de diverses façons au cours des quarante dernières années et examine les défis que ces identités multiples posent pour assurer sa reconnaissance professionnelle.

Playwork is an alternative approach to working with children developed by the UK adventure playground pioneers after the Second World War. Despite its rich history and current professional status in the UK, little accurate history is known about how the practice of playwork developed from the work on adventure playgrounds. This article describes the historical development of playwork from a community of practice to a national profession and now international movement. It outlines how playwork has been variously defined in the last forty years and discusses the challenges that these multiple identities pose in securing professional recognition of playwork on a global scale.

\section{INDEX}

Keywords : adventure playgrounds, playwork, play, out-of-school childcare, professional identity

Mots-clés : terrains d'aventure, playwork, jeu, accueils hors scolaires, identité professionnelle

\section{AUTEURS}

\section{SHELLY NEWSTEAD}

University College London

\section{TRADUCTEUR_DESCRIPTION}

\section{BAPTISTE BESSE-PATIN (TRADUCTION)}

Experice, Université Paris 13

\section{NATHALIE ROUCOUS (TRADUCTION)}

Experice, Université Paris 13 